\title{
Two-Fluid Model of the Solar Corona
}

\author{
by \\ J. W. Knight \\ C. E. Newman \\ P. A. Sturrock
}

March 1974

SUIPR Report No. 566

National Aeronautics and Space Administration Grant NGL 05-020-272

PUASA-CR-138613) SOLAB CORCNA (StanfOR-FLUID MODEL $\$ 4.00$

(stanford Univ.)

$12 \mathrm{p} \mathrm{HC}$ CSCL $03 B$ 


\section{TWO-FLUID MODEL OF THE SOLAR CORONA}

by

J.W. Knight, C.E. Newman and P.A. Sturrock

National Aeronautics and Space Administration

Grant NGL 05-020-272

SUIPR Report No. 566

March 1974

Institute for Plasma Research

Stanford University

Stanford, California 
TWO-FLUID MODEL OF THE SOLAR CORONA

\author{
by \\ J.W. Knight, C.E. Newman and P.A. Sturrock \\ Institute for Plasma Research \\ Stanford University \\ Stanford, California
}

\begin{abstract}
A simple model of the lower corona which allows for a possible difference in the electron and proton temperatures is analyzed. With the introduction of a phenomenological heating term, temperature and density profiles are calculated for several different cases. It is found that, under certain circumstances, the electron and proton temperatures may differ significantly.
\end{abstract}

PRECEOING PAGE BLANK NOT FILMED 


\section{INTRODUCTION}

It is generally believed that the corona is heated by a flux of non-thermal energy which originates in the photosphere and propagates through the chromosphere, transition region, and possibly part of the corona. D'Angelo (1969) suggests that if this non-thermal flux is in the form of ion acoustic waves, Landau damping of these waves might contribute significantly to the heating of the corona. D'Angelo also noted that if this were the case, preferential heating of the protons could cause the proton temperature to be higher than the electron temperature.

There are several interdependent aspects of the problem of coronal heating. These include the propagation of non-thermal energy, the dissipation of this energy, and the effect of this dissipation on the temperature and density structure of the corona. In this article, we shall be concerned only with the last topic, and we shall consider explicitly the possibility that a substantial fraction of the dissipated energy preferentially heats the tons.

\section{ANALYSIS}

We adopt a simple planar model of the corona in which all quantities are uniform in the horizontal coordinates and in which the gravitational acceleration is independent of the vertical coordinate $(z)$. The composition is taken to be that of fully ionized hydrogen gas. Radiation losses and magnetic flelds are neglected. Since we are not treating the problem of dissipation at this time, a phenomenological heating term is introduced. We suppose that all of the dissipation occurs in a narrow region, of width of order 10,000 kilometers, centered at a height of 15,000 kilometers 
above the base of our model, which we take to be the level at which $T=10^{6} \mathrm{o}$. The particular form adopted for the heating function $Q\left(\mathrm{erg} \mathrm{cm}^{-3} \mathrm{~s}^{-1}\right)$ is

$$
Q(z)=Q_{0} \sin ^{2}\left(\frac{\pi\left[z-z_{1}\right]}{z_{w}}\right), \quad z_{1} \leq z \leq z_{1}+z_{w}
$$

where $z_{1}=z_{w}=10,000 \mathrm{~km} . Q=0$ outside the range given in equation (1). We further assume that a fraction $f$ of the total energy input goes to heating the protons, so that

$$
Q_{-}=\left(\begin{array}{ll}
1 & -f
\end{array}\right) \mathrm{Q}, \quad Q_{+}=f Q
$$

where $Q_{-}(z), Q_{+}(z)$ are the electron and proton heating functions, respectively.

The relevant equations will be taken from Braginskii (1965). The equations of hydrostatic balance are

$$
\begin{aligned}
& \frac{-d p_{+}}{d z}+e_{+} E-m_{+} n_{+}=0 \\
& \frac{-d p_{-}}{d z}-e_{-} E-m_{-} n_{-}=0,
\end{aligned}
$$

where the symbols have their usual meaning. The subscripts + and refer to protons and electrons, respectively. We may set $n_{+} \approx n_{-}=n$ in the above equations (but not in Poisson's equation). Then, neglecting $\mathrm{m}_{-}$in comparison with $\mathrm{m}_{+}$and summing the above equations, we obtain

$$
\frac{\mathrm{d}}{\mathrm{dz}}\left(\mathrm{nk}\left[\mathrm{T}_{+}+\mathrm{T}_{-}\right]\right)+\mathrm{nm}_{+} \mathrm{g}=0 \text {. }
$$

Two heat equations may be expressed as

$$
\frac{d}{d z}\left(x_{+} \frac{d T+}{d z}\right)+Q_{+}-\frac{3}{2} \nu_{E} n k\left(T_{+}-T_{-}\right)=0
$$

and 


$$
\frac{d}{d z}\left(n_{-} \frac{d T-}{d z}\right)+Q_{-}+\frac{3}{2} \nu_{E} n k\left(T_{+}-T_{-}\right)=0 .
$$

The thermal conduction coefficients $x_{+}, x_{-}$and the heat-exchange coefficient ${ }^{\prime}$ E are given by

$n_{-}=10^{-4.73} \lambda^{-1} \mathrm{~T}_{-}^{5 / 2}, n_{+}=10^{-6.12} \lambda^{-1} \mathrm{~T}_{+}^{5 / 2}, \nu_{\mathrm{E}}=10^{-2.41} \lambda^{-1} \mathrm{n} \mathrm{T}_{-}^{-3 / 2}$,

where $\lambda$ (the Coulomb logarithm) can be expressed with sufficient accuracy as

$$
\lambda=15.9-1.15 \log \mathrm{n}+2.3 \log \mathrm{T}_{-} .
$$

For the ranges of density and temperature of interest, we may with sufficient approximation adopt $\lambda=20$. Then the coefficients in equation (8) may be expressed as

$$
x_{-}=\frac{7}{2} \mathrm{~K}_{-} \mathrm{T}_{-}^{5 / 2}, \quad x_{+}=\frac{7}{2} \mathrm{~K}_{+} \mathrm{T}_{+}^{5 / 2}, \quad v_{\mathrm{E}}=\frac{2}{3} \mathrm{Ak}^{-1} \mathrm{n} \mathrm{T}_{-}^{3 / 2}
$$

where. $\mathrm{K}_{-}=10^{-6.57}, \mathrm{~K}_{+}=10^{7.96}$ and $\mathrm{A}=10^{-16.79}$.

With these substitutions, equations (6) and (7) become

$$
\begin{aligned}
& \frac{\mathrm{d}^{2} \mathrm{~T}_{-}^{7 / 2}}{\mathrm{dz}}=-\mathrm{K}_{-}^{-1} \mathrm{Q}_{-}-\mathrm{AK}_{-}^{-1} \mathrm{n}^{2} \mathrm{~T}_{-}^{-3 / 2}\left(\mathrm{~T}_{+}-\mathrm{T}_{-}\right), \\
& \frac{\mathrm{d}^{2} \mathrm{~T}_{+}^{7 / 2}}{\mathrm{dz}}=-\mathrm{K}_{+}^{-1} \mathrm{Q}_{+}+\mathrm{AK}_{+}^{-1} \mathrm{n}^{2} \mathrm{~T}_{-}^{-3 / 2}\left(\mathrm{~T}_{+}-\mathrm{T}_{-}\right),
\end{aligned}
$$

from which we see that

$$
\frac{\mathrm{d}^{2}}{\mathrm{dz}^{2}}\left(\mathrm{~K}_{-} \mathrm{T}_{-}^{7 / 2}+\mathrm{K}_{+} \mathrm{T}_{+}^{7 / 2}\right)=-\mathrm{Q} .
$$

Equation (5) now becomes

$$
\frac{1}{n} \frac{d n}{d z}=-\left(T_{-}+T_{+}\right)^{-1}\left(C+\frac{d}{d z}\left[T_{-}+T_{+}\right]\right)
$$

where 


$$
\mathrm{C}=\mathrm{m}_{+} \mathrm{gk}^{-1} \approx 10^{-3.48} \text {. }
$$

\section{III . RESULTS}

our aim is to calculate $n(z), T_{-}(z)$ and $T_{+}(z)$ for specified forms of $Q_{-}(z)$ and $Q_{+}(z)$, and for given boundary conditions. One of the five required boundary conditions is as follows,

$$
\mathrm{n}=\mathrm{n}_{\mathrm{o}} \text { at }, \mathrm{z}=\mathrm{0}
$$

Since equation (13) is immediately integrable, it is convenient to express two of the conditions as follows,

$$
\mathrm{K}_{-} \mathrm{T}_{-}^{7 / 2}+\mathrm{K}_{+} \mathrm{T}_{+}^{7 / 2}=\left(\mathrm{K}_{-}+\mathrm{K}_{+}\right) \mathrm{T}_{0}^{7 / 2} \text { at } \mathrm{z}=0
$$

and

$$
\frac{d}{d z}\left(K_{-} T_{-}^{7 / 2}+K_{+} T^{7 / 2}\right)=\int_{Z_{I}}^{z_{1}+z w} Q d x \equiv F
$$

Equation (18) implies that the entire energy input flows back down as a heat flux. The remaining conditions are taken to be the following:

$$
\begin{aligned}
& \mathrm{T}_{+}-\mathrm{T}_{-} \rightarrow 0, \mathrm{z} \rightarrow-\infty, \\
& \mathrm{T}_{+}-\mathrm{T}_{-} \rightarrow 0, \mathrm{z} \rightarrow \infty,
\end{aligned}
$$

In selecting the boundary conditions at the base of the model, we have considered estimates for the product nT in the transition region. Athay (1965) estimated this quantity to be in the range of $(3.7-6.0) \times 10^{14}$; later, Athay (1969) made the estimate $6 \times 10^{14}$; Moore and Fung (1972) adopt the range $(5-15.1) \times 10^{14}$. From these estimates, we have chosen the values $\log n_{0}=8.50,8.78,9.00$ at $\mathrm{T}=10^{6}$. We also consider

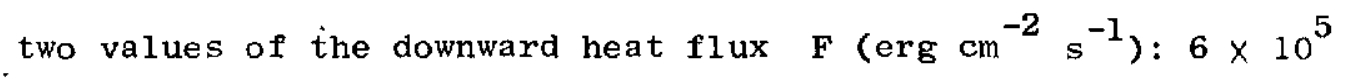


(Dupree and Goldberg 1967), and $10^{6}$ (Moore 1972).

The results of our calculations are displayed in Figures 1 and 2 . We see from Figure 1 that an increase in $n_{0}$ leads to a similar increase in $n(z)$, so that the profile is insensitive to $n_{0}$. An increase of $\mathrm{n}_{\mathrm{o}}$ hardly affects $\mathrm{T}_{-}(\mathrm{z})$, but it leads to a reduction of $\mathrm{T}_{+}(\mathrm{z})$ since it tightens the coupling between the two species. (Note, however, that the asymptotic value of $T_{+}$, as $z \rightarrow \infty$, is not significantly affected by $\mathrm{n}_{0}$ since $\mathrm{T}_{+} \rightarrow \mathrm{T}_{-}$in this limit.)

Figure 1 also shows that, if $n_{0}$ and $F$ are fixed, an increase in $f$ leads to an increase in $T_{+}$in the heating region and to a slight reduction in $n$ in this region. The decrease in density tends to offset the increase in pressure due to the increase in proton temperature. The electron temperature is not significantly effected by a change in $f$. We see from Figure 2 that, for fixed $F$ and $f$, an increase in $n_{0}$ leads to an increase of $n(z)$ and a reduction in $T_{+}(z)$, as noted above, but this diagram shows that there is a slight increase in $T_{-}(z)$ in the heating region. A comparison of Figures 1 and 2 shows that an increase in $F$, for fixed $n_{0}, f$, leads to increases in $n(z), T_{-}(z)$ and $T_{+}(z)$.

It is notable that, if $n_{0}$ is as small as $10^{8.5}, T_{+}$may exceed $\mathrm{T}_{-}$, in the heating region, by up to $70 \%$.

The above calculations show that, if the non-thermal energy goes primarily into heating the proton gas, the proton temperature may be substantially higher than the electron temperature in the heating region of the corona. Hence one would need to be cautious in inferring the coronal structure from observational data, since some observations (such as radio-frequency brightness and coronal line strengths) depend upon electron temperature, other observations (such as corona1-line widths) 
depend upon the ion temperature, and some observations (such as the variation of density with radius) depend upon a combination of the temperatures.

In order to pursue this question, it is essential to investigate in more detail the heating of the solar corona, to determine in particular the allocation of energy between the electron gas and the proton gas.

\section{ACKNOWLEDGEMENT}

This work was supported by the National Aeronautics and Space Administration under Grant NGL 05-020-272, 


\section{REFERENCES}

Athay, R.G., 1965, Ap. J. 142, 755 .

Athay, R.G., 1969, Solar Phys. 9, 51.

Braginskii, S.I., 1965, Reviews of Plasma Phys. 1, 205.

D'Angelo, N., 1969, Solar Phys. 7, 321.

Dupree, A.K. and Goldberg, L., 1967, Solar Phys. 1, 229.

Moore, R.L., 1972, Stanford University Institute for Plasma Rese arch Report No. 463 .

Moore, R.L. and Fung, P.C.W., 1972, Solar Phys. 23, 78. 


\section{FIGURE CAPTIONS}

Figure 1. Temperature and density profiles for a total energy input of $1.0 \times 10^{6} \mathrm{erg} \mathrm{cm}^{-2} \mathrm{sec}^{-1}$. Case I corresponds to an initial density of $10^{9} \mathrm{~cm}^{-3}$ and $f=1$. Case $I I$ also corresponds to an initial density of $10^{9} \mathrm{~cm}^{-3}$ but with $f=0.5$. Case III corresponds to an initial density of $10^{8.78} \mathrm{~cm}^{-3}$ and $f=1$. The T_ profiles are indistinguishable on this scale.

Figure 2. Temperature and density profiles for a total energy input of $6 \times 10^{5} \mathrm{erg} \mathrm{cm}^{-2} \mathrm{sec}^{-1}$ and $\mathrm{f}=1$. Case I corresponds to an initial density of $10^{9} \mathrm{~cm}^{-3}$. Case II corresponds to an initial density of $10^{8.78} \mathrm{~cm}^{-3}$. (The $T_{-}$curves for these first two cases are indistinguishable.) Case III corresponds to an initial density of $10^{8.5} \mathrm{~cm}^{-3}$. 
Figure 1

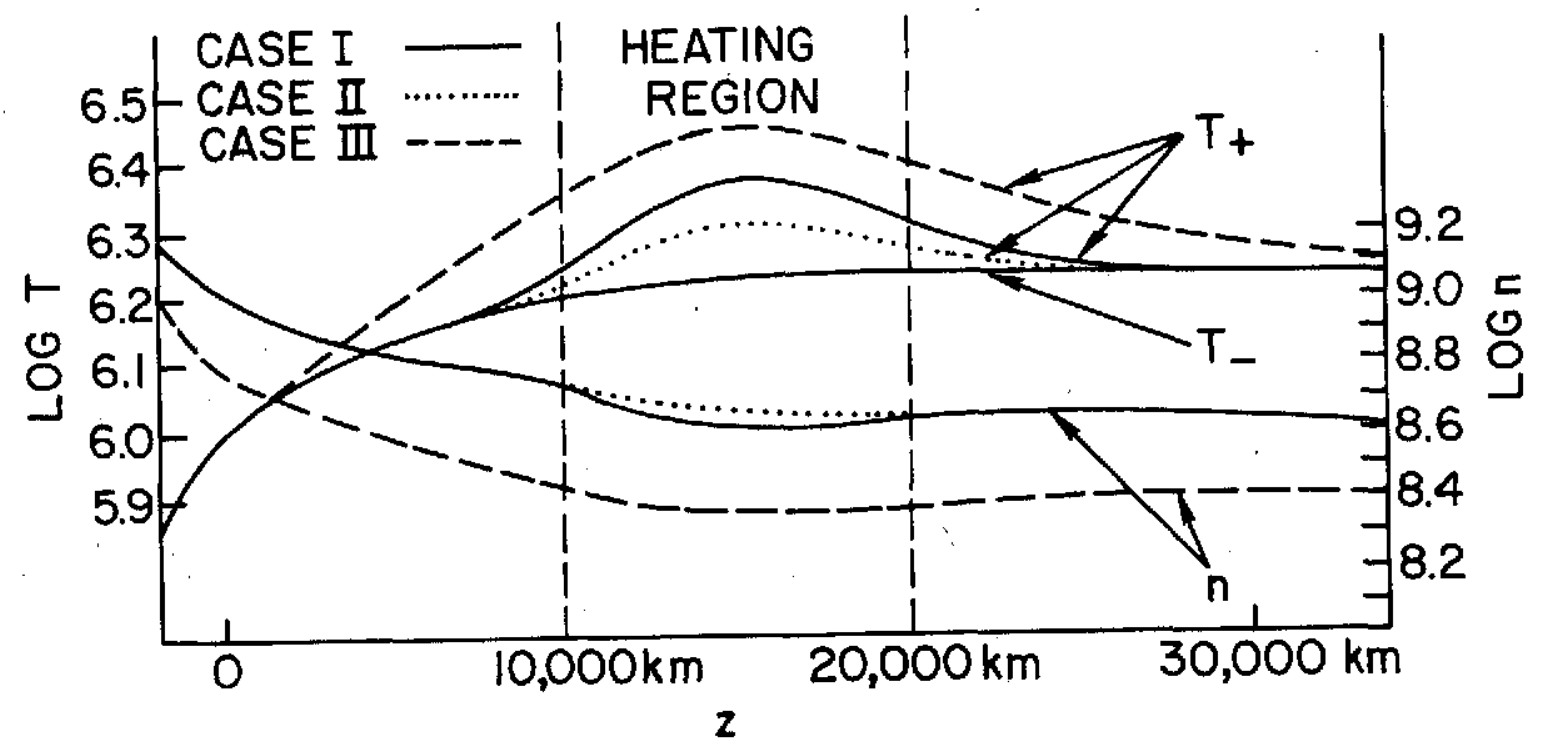

Figure 2

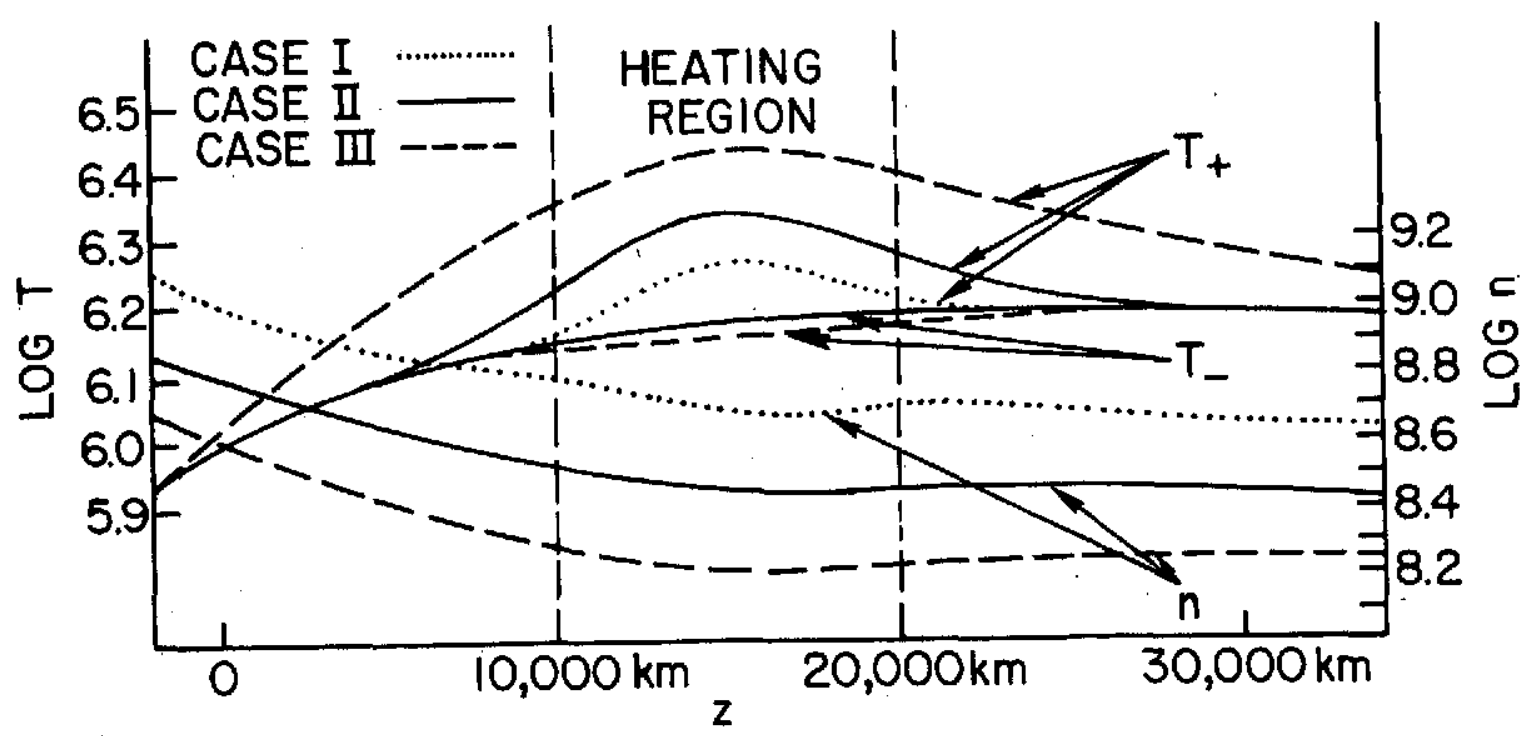

